03.1

\title{
Смещения предела устойчивости течения при модуляции скорости вращения
}

\author{
(С) Д.Ю. Жиленко, О.Э. Кривоносова \\ Институт механики Московского государственного университета им. М.В. Ломоносова, Москва, Россия \\ E-mail: jilenko@imec.msu.ru
}

Поступило в Редакцию 19 марта 2020 г.

В окончательной редакции 19 марта 2020 г.

Принято к публикации 23 марта 2020 г.

\begin{abstract}
Экспериментально исследованы возможности управления положением предела устойчивости в сферическом течении Куэтта. Скорость вращения внутренней сферы периодически изменяется относительно ненулевого среднего значения, внешняя сфера неподвижна. Такая же, как и при стационарном вращении, неустойчивость в виде бегущих азимутальных волн вызывается повышением средней скорости вращения. Проведены измерения скорости течения лазерным доплеровским анемометром. Показано, что при приближении частоты модуляции к собственной частоте линейной моды в зависимости от величины амплитуды возможны как дестабилизация течения, так и его стабилизация.
\end{abstract}

Ключевые слова: сферическое течение Куэтта, вращение, неустойчивость.

DOI: 10.21883/PJTF.2020.12.49522.18295

Течения с неравномерным во времени вращением встречаются во многих практических приложениях: например, при неустойчивости плазмы в сферическом токамаке [1] и при интенсификации фильтрации жидкости [2]. В течениях в сферических слоях, которые рассматриваются в настоящей работе, периодическая модуляция скорости вращения может приводить к частичному [3] и полному подавлению турбулентности [4], что может использоваться в технологиях обработки жидких металлов и расплавов [5]. Подавление турбулентности наблюдалось и для течений в трубах в случае изменения профилей осевой скорости [6]. В то же время результаты исследований устойчивости течений под влиянием периодических во времени граничных условий приводят к различным выводам. Так, течение Тэйлора-Куэтта в цилиндрическом зазоре становится неустойчивым при меньших, чем при стационарном вращении, числах Рейнольдса как при модуляции скорости вращения [7], так и при ее ступенчатом изменении [8,9]. Наоборот, в плоском течении Куэтта в случае осцилляций стенок в направлении поперек течения обнаружены его стабилизация и затягивание перехода к турбулентности [10]. Целью настоящей работы является экспериментальное исследование влияния осцилляций скорости вращения на величину и направление сдвига предела устойчивости в сферическом течении Куэтта - течении вязкой несжимаемой жидкости, формирующемся в сферическом зазоре между коаксиально расположенными сферами под действием вращения одной из них. Сферический зазор в эксперименте формируется двумя прозрачными сферами, каждая из которых имеет свой привод, и заполнен силиконовым маслом, вязкость которого при температуре $22^{\circ} \mathrm{C}$ составляет $v \approx 5 \cdot 10^{-5} \mathrm{~m}^{2} / \mathrm{s}$. В силиконовое масло добавлена алюминиевая пудра с целью визуализации течений в слое. Радиус внутренней сферы $r=0.075 \mathrm{~m}$, радиус внешней $r_{\mathrm{o}}=0.15 \mathrm{~m}$, они размещены в термостате с силиконовым маслом с целью сохранения сферичности границ и стабилизации температуры в слое. Измерение температуры проводилось датчиком, расположенным на внешней сфере. Температура поддерживалась постоянной с погрешностью не более $0.05^{\circ} \mathrm{C}$. Измерения азимутальной компоненты скорости течения $u_{\varphi}$ проводились лазерным анемометром вблизи внешней сферы, в точке, удаленной от плоскости экватора на расстояние $0.078 \mathrm{~m}$ и от оси вращения на расстояние $0.105 \mathrm{~m}$. Внешняя сфера неподвижна, скорость внутренней сферы $\Omega_{1}(t)$ периодически изменялась относительно ненулевого среднего значения $\Omega_{0}$

$$
\Omega_{1}(t)=\Omega_{0}+A \sin (2 \pi f t+\varphi) .
$$

Здесь $A$ и $f$ - соответственно амплитуда и частота, величины которых сохранялись системой управления постоянными с погрешностью не более $0.05 \%$ от заданных значений.

В отсутствие колебаний $(A=0)$ течение стационарно и симметрично относительно оси вращения и экваториальной плоскости при числах Рейнольдса [11]:

$$
\operatorname{Re}_{1}=\left(\Omega_{0} r^{2}\right) / v<\operatorname{Re}_{c}=460 \pm 2
$$

где $\operatorname{Re}_{c}$ - предел устойчивости течения в рассматриваемом сферическом слое при стационарном вращении. Потеря устойчивости в этом слое происходит мягко (без гистерезиса), симметрии теряются, во вторичном течении образуются азимутальные волны с волновыми числами $m=3$ или $m=4$ [11]. Вблизи предела устойчивости собственные частоты линейных мод составляют для $m=3 \quad f_{3}=0.3-0.32 \mathrm{~Hz}$ и для $m=4$ 
$f_{4}=0.4-0.43 \mathrm{~Hz}$. Амплитуды колебаний скорости вторичного течения при небольшой надкритичности пропорциональны, согласно [12], выражению $\left(\operatorname{Re}_{1}-\operatorname{Re}_{c}\right)^{1 / 2}$, что экспериментально подтверждено и в случае присутствия незначительного (не более $1 \%$ ) шума в сигнале скорости вращения внутренней сферы [13]. Этот результат позволяет использовать приведенное выше выражение для определения малых по величине изменений положения предела устойчивости под действием периодических колебаний скорости вращения внутренней сферы. Эксперимент проводился следующим образом: сначала при $\operatorname{Re}_{1}=0.8 \operatorname{Re}_{c}$ устанавливалось основное течение, затем включалась модуляция с неизменными в ходе каждого эксперимента величинами $A$ и $f$. Далее величина $\Omega_{0}$ ступенчатым образом квазистатически повышалась до потери течением устойчивости, момент наступления которой определялся по визуализации. Затем происходило ступенчатое снижение $\Omega_{0}$, и на участках с постоянными значениями $\Omega_{0}$ проводились измерения $u_{\varphi}$. Так же как в [4,13], амплитуда колебаний скорости течения на реализующейся в эксперименте моде вторичного течения определялась с использованием преобразования Гильберта (НТ)

$$
A_{f}=\left|u_{f}(t)+i \mathrm{HT}\left(u_{f}(t)\right)\right| .
$$

Здесь $u_{f}(t)$ предварительно выделялась из $u_{\varphi}(t)$ фильтрацией в полосе частот $f_{3} \pm \Delta f$ для $m=3$ и $f_{4} \pm \Delta f$ для $m=4, \Delta f=0.005-0.010 \mathrm{~Hz}$. Для определения положения предела устойчивости полученные зависимости $A_{f}$ от $\operatorname{Re}_{1}$ аппроксимировались указанным выше выражением. Необходимость аппроксимации вызвана увеличением времени переходных процессов при $\left(\operatorname{Re}_{1}-\operatorname{Re}_{c}\right) \rightarrow 0$, что исключает возможность достоверного определения малых величин $A_{f}$. Сравнение с аналогичной зависимостью $A_{f}$ от $\mathrm{Re}_{1}$, полученной в отсутствие колебаний скорости вращения, позволяет определить направление и величину изменения положения предела устойчивости.

Результаты визуализации течений при $A \neq 0$ показали, что до потери устойчивости периодическое во времени (сформировавшееся после наложения модуляции на скорость внутренней сферы) течение остается симметричным относительно экватора. Однако модуляция скорости вращения вызывает изменение профиля азимутальной скорости, что обусловлено эффектами нелинейности и вязкости [14]. На рис. 1, a показана зависимость относительного изменения скорости течения $U$ от относительной амплитуды модуляции $\varepsilon$ до потери устойчивости при $\operatorname{Re}_{1} / \operatorname{Re}_{c}=0.95 ; U=u_{\varphi 1} / u_{\varphi 0}$, $\varepsilon=\left(\left(A r^{2}\right) / v\right) / \operatorname{Re}_{c}, u_{\varphi 1}$ и $u_{\varphi 0}$ - азимутальная скорость течения при $A \neq 0$ и $A=0$ соответственно. Скорость течения монотонно возрастает с увеличением $\varepsilon$ при всех $f$, в исследуемом диапазоне $\varepsilon$ наибольшие отличия скорости от случая стационарного течения обнаружены при $f=0.5 \mathrm{~Hz}$. Неустойчивость течения при $A \neq 0$ сопровождается формированием такой же, как и в случае

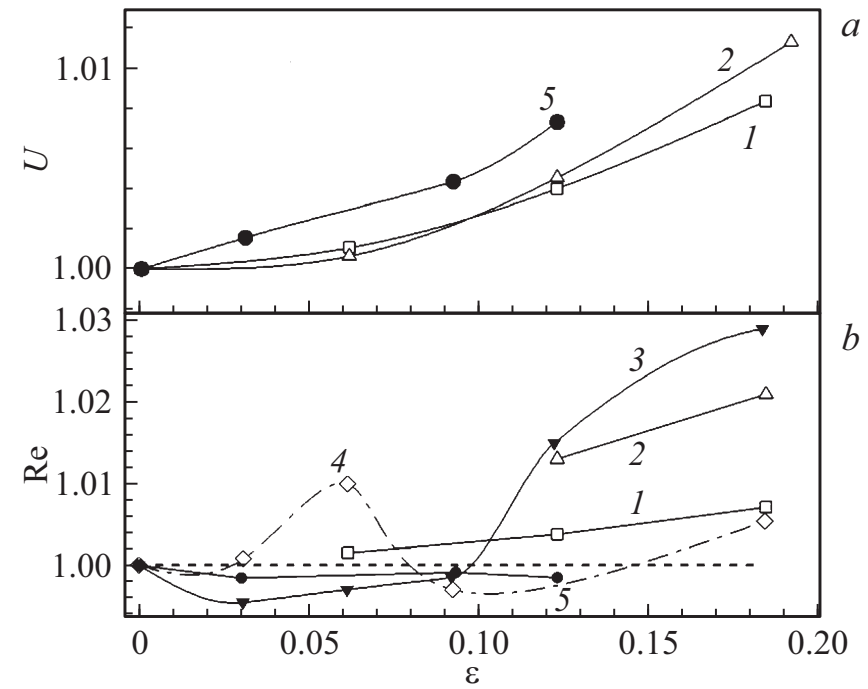

Рис. 1. Полученные в эксперименте отношения скоростей $U(a)$ и чисел Рейнольдса $\operatorname{Re}(b)$ в зависимости от относительной амплитуды модуляции $\varepsilon . f, \mathrm{~Hz}: 1-0.125,2-$ $0.25,3-0.28,4-0.3,5-0.5$. Горизонтальная штриховая линия соответствует условию $\mathrm{Re}=1$.

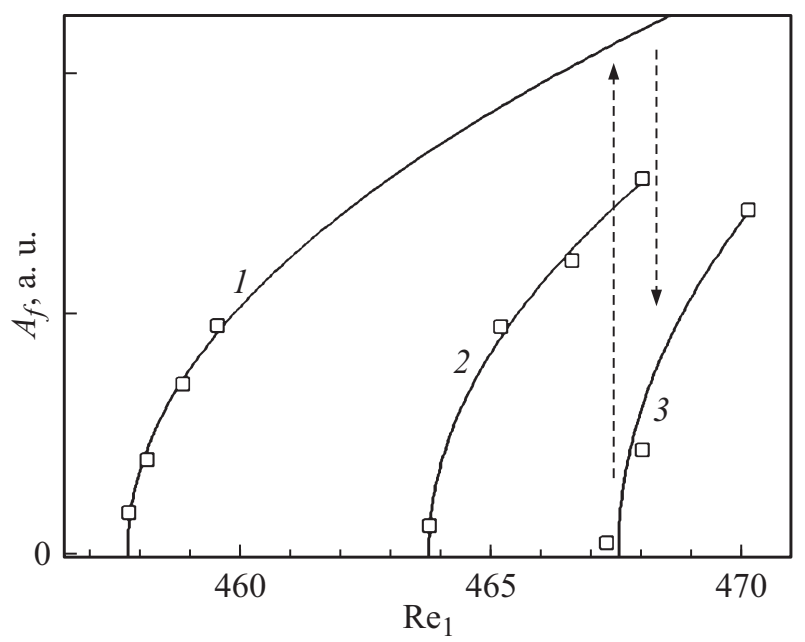

Рис. 2. Зависимость амплитуды $A_{f}$ от $\operatorname{Re}_{1}$ при $f=0.25 \mathrm{~Hz}$ при различных значениях $\varepsilon$. $\varepsilon=0(1), 0.123$ (2) и 0.1845 (3).

$A=0$, структуры, а именно азимутальных волн с волновыми числами $m=3$ или $m=4$. Но величина числа Рейнольдса, соответствующего потере устойчивости в этом случае $\left(\operatorname{Re}_{c 1}\right)$, может отличаться от $\operatorname{Re}_{c}$ при $A=0$, причем и величина, и направление смещений предела устойчивости зависят от $A$ и $f$. На рис. $1, b$ представлены зависимости относительного изменения положения предела устойчивости $\operatorname{Re}=\operatorname{Re}_{c 1} / \operatorname{Re}_{c}$ от относительной амплитуды модуляции $\varepsilon$. $\operatorname{Re}<1$ означает уменьшение $\operatorname{Re}_{c 1}$ по сравнению со случаем стационарного вращения, течение при этом дестабилизируется модуляцией скорости вращения, а при $\operatorname{Re}>1$ течение стабилизируется. Полученные результаты свидетельствуют о том, что при 


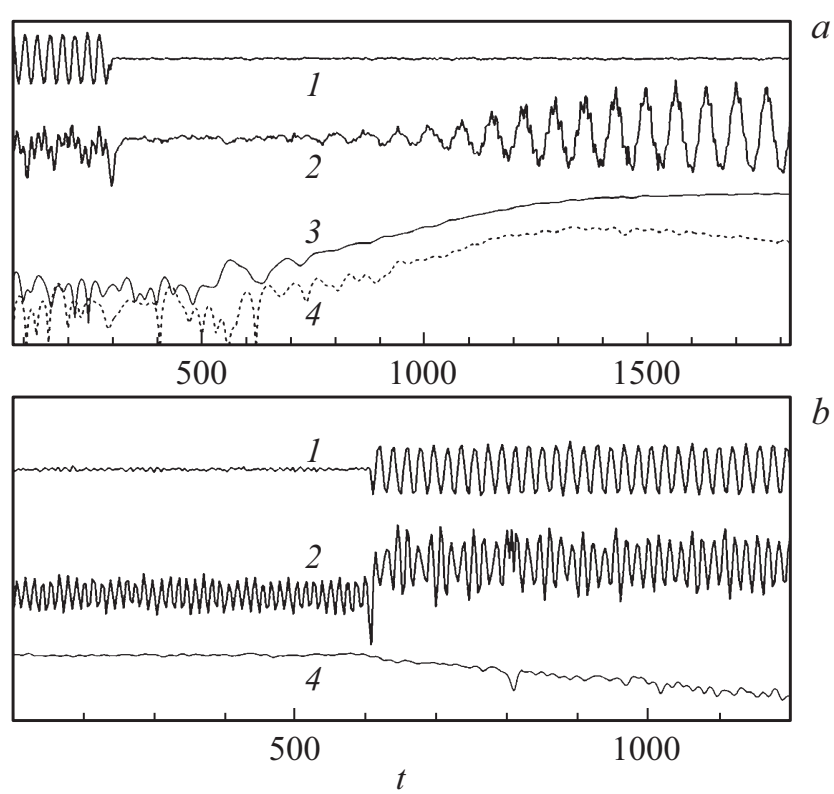

Рис. 3. Изменение во времени $t$ скорости внутренней сферы (1), измеряемой скорости течения (2), амплитуды $A_{f}$ мод $m=3$ (3) и $m=4$ (4). a- выключение модуляции при $\mathrm{Re}=1.0207, b-$ включение модуляции при $\mathrm{Re}=1.0047$. На части $b A_{f}(m=3)$ близка к нулю и поэтому не показана.

величинах $f$, меньших, чем собственные частоты линейных мод $f_{3,4}$ (рис. $1, b$, кривые 1 и 2), течение стабилизируется, $\operatorname{Re}$ монотонно возрастает с увеличением $\varepsilon$. При $f>f_{3,4}$ возможна незначительная дестабилизация (рис. $1, b$, кривая 5). Наиболее сложная немонотонная зависимость $\operatorname{Re}$ от $\varepsilon$ наблюдается при приближении $f$ к частоте $f_{3}$ (рис. $1, b$, кривые 3 и 4). В случае $f=0.28 \mathrm{~Hz}$ (рис. $1, b$, кривая 3) при $\varepsilon<0.1$ течение дестабилизируется, тогда как при $\varepsilon>0.1$ наблюдается стабилизация. При $f=f_{3}$ (рис. $1, b$, кривая 4) зависимость $\operatorname{Re}$ от $\varepsilon$ усложняется: течение испытывает два цикла дестабилизация-стабилизация при увеличении $\varepsilon$. Подобная восприимчивость к возмущениям, частота которых соответствует собственной частоте когерентных структур, отмечена и в случае подавления турбулентности в сферических слоях [4]. Показанные экспериментально в данной работе возможности стабилизации течения под действием модуляции скорости вращения не соответствуют результатам, полученным ранее для цилиндрического течения Тэйлора-Куэтта, где, как отмечено выше, наблюдалась только дестабилизация [7]. Для подтверждения возможности стабилизации течения в сферическом слое рассмотрим зависимость $A_{f}$ от $\mathrm{Re}_{1}$ при различных $\varepsilon$ (рис. 2). Переход от $\varepsilon>0$ к $\varepsilon=0$ (выключение модуляции) при постоянной величине $\operatorname{Re}_{1}$ должен приводить к увеличению $A_{f}$, а переход в обратном направлении должен приводить к снижению $A_{f}$ (вертикальные стрелки на рис. 2). На рис. 3 показаны такие переходы при $f=0.25 \mathrm{~Hz}$ и $\varepsilon=0.1845$. При выключении модуляции скорости вращения (рис. 3,a) пульсации $u_{\varphi}$ сначала затухают, но по мере развития неустойчивости и формирования бегущей азимутальной волны возрастают, при этом начинается экспоненциальный рост обеих возможных линейных мод. Мода $m=4$ проходит через максимум и начинает затухать, а мода $m=3$ выходит на постоянное значение. На рис. $3, b$ показан случай перехода от $\varepsilon=0$ к $\varepsilon>0$. До включения модуляции в течении присутствует только мода $m=4$. После включения модуляции амплитуда этой моды затухает, хотя амплитуда пульсаций $u_{\varphi}$ при этом увеличивается. Это означает, что течение начинает перестраиваться в периодическое, аналогичное периодическому течению до потери устойчивости. Все эти результаты полностью соответствуют рис. 2 и являются дополнительным подтверждением возможности стабилизации течения.

Таким образом, установлено, что во вращающемся сферическом слое величина и направление сдвига предела устойчивости, происходящего под действием периодических колебаний скорости вращения, определяются в основном частотой модуляции. При частотах модуляции, меньших, чем собственные частоты линейных мод, течение стабилизируется: его предел устойчивости выше, чем при стационарном вращении, и растет с увеличением амплитуды модуляции. При частоте модуляции, превышающей собственные частоты линейных мод, течение дестабилизируется, и уменьшение предела устойчивости по сравнению со стационарным вращением слабо зависит от амплитуды. При частотах модуляции, приближающихся к частоте линейной моды, направление и величина сдвига предела устойчивости немонотонно зависят от амплитуды. При меньших величинах амплитуды возможна дестабилизация течения, а при больших амплитудах — стабилизация.

\section{Финансирование работы}

Работа выполнена при частичной финансовой поддержке Российского фонда фундаментальных исследований (проекты № 18-08-00074 и 19-05-00028).

\section{Конфликт интересов}

Авторы заявляют, что у них нет конфликта интересов.

\section{Список литературы}

[1] Буланин В.В., Гусев В.К., Курскиев Г.С., Минаев В.Б., Патров М.И., Петров А.В., Петров М.А., Петров Ю.В., Тельнова А.Ю., Яшин А.Ю. // Письма в ЖТФ. 2017. Т. 43. B. 23. C. $40-47$.

[2] Jaffrin M. // Annu. Rev. Fluid Mech. 2012. V. 44. P. 77-96.

[3] Жиленко Д.Ю., Кривоносова О.Э. // Письма в ЖТФ. 2017. T. 43. В. 10. С. 87-94.

[4] Жиленко Д.Ю., Кривоносова О.Э. // Письма в ЖТФ. 2019. T. 45. B. 17. C. $20-23$

[5] Нашельский А.Я. Производство полупроводниковых материалов. М.: Металлургия, 1989. 272 с. 
[6] Marensi E., Willis A.P., Kerswell R.R. // J. Fluid Mech. 2019. V. 863. P. $850-875$.

[7] Feugaing C.M.G., Crumeyrolle O., Yang K.-S., Mutabazi I. // Eur. J. Mech. B. 2014. V. 44. P. 82-87.

[8] Ватажсин А.Б., Гуськов О.В. // Изв. РАН. Механика жидкости и газа. 2010. № 1. С. 45-56.

[9] Kaiser F., Frohnapfel B., Ostilla-Monico R., Kriegseis J., Rival D.E., Gatti D. // J. Fluid Mech. 2020. V. 885. P. A6.

[10] Rabin S.M.E., Caulfield C.P., Kerswell R.R. // J. Fluid Mech. 2014. V. 738. P. R1.

[11] Беляев Ю.Н., Яворская И.М. // Итоги науки и техники. Сер. Механика жидкости и газа. 1980. Т. 15. С. 3-80.

[12] Ландау Л.Д., Лившии, Е.М. Теоретическая физика. Т. 6. Гидродинамика. 3-е изд. М.: Наука, 1986. 736 с.

[13] Zhilenko D., Krivonosova O., Gritsevich M., Read P. // Chaos. 2018. V. 28. P. 053110.

[14] Le Bars M., Cebron D., Le Gal P. // Annu. Rev. Fluid Mech. 2015. V. 47. P. 163-193. 